\title{
Improved Clival Gate 4H-SiC MESFET with Recessed Drain Drift Region and Recessed P-Buffer Layer
}

\author{
Hujun Jia, Peimiao Ma*, Yehui Luo, Ding Xing, Hang Zhang, Zhijiao Wang and Qiuyuan Wu \\ Key lab. Of Wide Band Gap Semiconductor Materials and Devices, Xidian University, 710071 Xi' an, China \\ ${ }^{*}$ Corresponding author
}

\begin{abstract}
An improved clival gate 4H-SiC MESFET with recessed drain drift region and recessed p-buffer layer (RDRPCG MESFET) was proposed in this paper. The key improvement in this paper is the enhancement of the drain current and the breakdown voltage. The recessed drain drift region and recessed p-buffer layer are introduced in the proposed structure to ensure the rise of the drain current and the breakdown voltage simultaneously. DC and RF characteristics are simulated and compared to the clival gate $4 \mathrm{H}$-SiC MESFET (CG MESFET). The numerical simulated results show that the breakdown voltage is $116.3 \mathrm{~V}$ compared to $76.6 \mathrm{~V}$ of CG MESFET, which is about $51.8 \%$ larger than that of CG MESFET. There is an $11.9 \%$ increase in drain saturation current of RDRP-CG MESFET compared with CG MESFET. Thus, the maximum output power density is about $\mathbf{7 0} \%$ larger than that of CG MESFET, which is due to the increase in drain saturation current and breakdown voltage. There is a slight decrease in cut-off frequency in the proposed structure.
\end{abstract}

Keywords-component; 4H-SiC MESFET; DC characteristics; RF characteristics

\section{INTRODUCTION}

With the development of wireless communication, the requirements for transceiver system become more and more rigorous, especially in efficiency. As the key component for transmitter, the power amplifier consumes a lot of energy, which results in a large decrease in efficiency. Improving the efficiency of power amplifier is a priority for RF system. The traditional way is at the cost of conduction angle, which is not suitable for wireless communication. Compared to the traditional way, an amplifier named switching power amplifier is introduced to enhance efficiency because of the drop in power consumption by reducing the product of voltage and current. Due to the high efficiency, class-E power amplifier is widely applied in RF system. The extremely strict requirements for device make $\mathrm{SiC}$ MESFET be the best candidate for class-E power amplifier due to its superior properties, which is proved by some researchers [1], such as high thermal conductivity, high power density, strong endurance to high temperature. Some new structures [2-5] are proposed to improve the performance of SiC MESFET, which make SiC MESFET sustain more superior performance for RF system, such as double recessed MESFET (DR MESFET), multiple recessed MESFET (MR MESFET), clival MESFET (CG MESFET). DR MESFET consists of lower and upper gates, which ensures the channel can be controlled by gate bias and allows for a large drain current, respectively. However, the structure gets a reduction in breakdown voltage because of the thick channel thickness. CG MESFET, a structure with clival gate, which is considered as a kind of MR MESFET with an infinite number of small steps, is proposed to obtain larger drain current and higher breakdown voltage simultaneously compared to DR MESFET. However, the breakdown voltage is not high enough for high power application.

Above all, it is a great challenge to ensure large drain current and high breakdown at the same time. In RF system, device usually needs endure high voltage. Therefore, a high breakdown voltage is not only benefit for the maximum output power density, but also for high efficiency high power RF system. For this end, an improved clival gate $4 \mathrm{H}-\mathrm{SiC}$ MESFET with recessed drain drift region and recessed $p$ buffer layer (RDRP-CG MESFET) is proposed in this paper. Recessed drain drift region can modulate the electric field in the channel and modify surface electric field distribution, and therefore will improve the breakdown voltage. To allow for a large drain current simultaneously, recessed p-buffer layer is introduced to maintain a thick channel. Simulated results indicate that the recessed drain drift region and the recessed pbuffer layer maintain a larger drain current and a higher breakdown voltage than those of CG MESFET. The characteristics of RDRP-CG MESFET are discussed in detail and compared with CG MESFET in the paper.

\section{DEVICE StRUcture}

Figure I(a) and Figure I(b) show the schematic crosssections of CG MESFET and RDRP-CG MESFET, respectively. Two structures have some uniform parameters to get a meaningful comparison: a high-purity semi-insulating substrate, a p-buffer with the thickness of $0.5 \mu \mathrm{m}$ and doping concentration of $1.4 \times 10^{15} \mathrm{~cm}^{-3}$, an n-channel with the thickness of $0.25 \mu \mathrm{m}$ and doping concentration of $3 \times 10^{17} \mathrm{~cm}^{-3}$,two cap layer with doping concentration of $1 \times 10^{20} \mathrm{~cm}^{-3}$, source-gate spacing $\mathrm{L}_{\mathrm{gs}}=0.5 \mu \mathrm{m}$, gate-drain spacing $\mathrm{L}_{\mathrm{gd}}=1.8 \mu \mathrm{m}$, the length of gate $\mathrm{L}_{\mathrm{g}}=0.7 \mu \mathrm{m}$, lower gate height $\mathrm{H} 1=0.06 \mu \mathrm{m}$, and the end point of clival gate is at the middle of the gate. There are some different parameters in FigureI(b), the height and length of recessed drain drift region formed by etching are $0.06 \mu \mathrm{m}$ and $0.5 \mu \mathrm{m}$, respectively, that means $\mathrm{H} 1=0.06 \mu \mathrm{m}, \mathrm{L}_{\mathrm{rd}}=0.5 \mu \mathrm{m}$. The depth and length of recessed p-buffer layer are $0.1 \mu \mathrm{m}$ and $0.5 \mu \mathrm{m}$. Nickel is chosen for gate Schottky contact with a work 
function of $5.1 \mathrm{eV}$. A temperature of $300 \mathrm{~K}$ is employed in simulations by default.

A 2D simulator named ISE-TCAD is applied for the simulation of characteristics of device with $4 \mathrm{H}-\mathrm{SiC}$ material parameters. The corresponding electric characteristics of the device are simulated mainly by solving a series of partial differential equations of mathematical physics, such as Poisson equation, drift-diffusion equation. All of the parameters in these equations must have physical models to match. The physical models include Avalanche for generation and recombination, Doping, Enormal and HighFieldSat for mobility and Incomplete for incomplete ionization of dopants [6], etc.
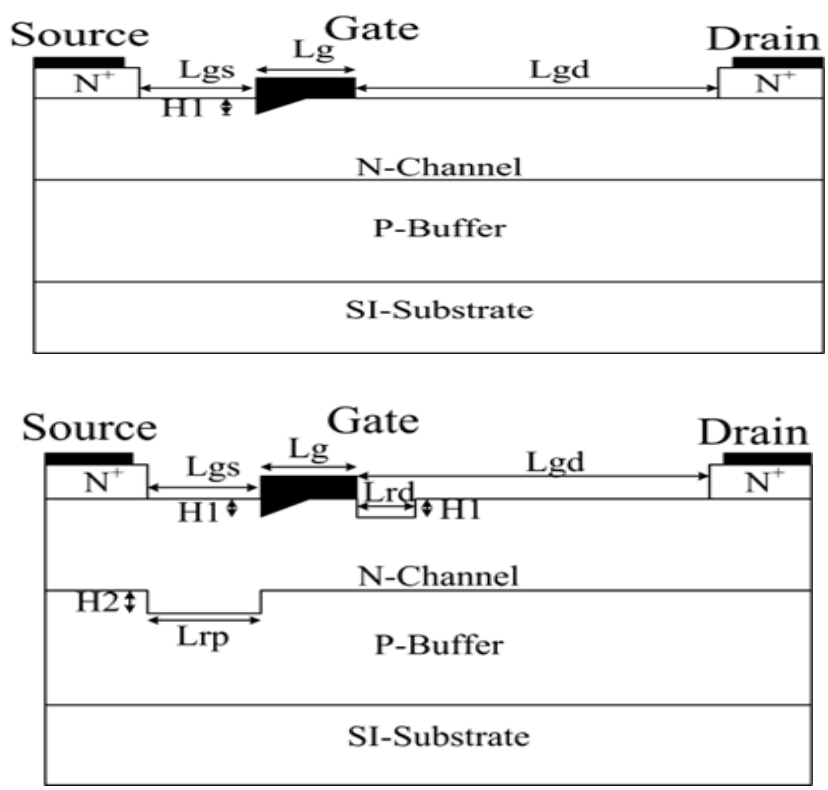

FIGURE I. SCHEMATIC CROSS-SECTIONS OF THE (A) CG MESFET AND (B) RDRP-CG MESFET STRUCTURES

\section{RESULTS AND DisCUSSION}

\section{A. DC Characteristics}

Figure II shows the simulated drain current $\left(I_{d}\right)$ versus drain-source voltage $\left(\mathrm{V}_{\mathrm{ds}}\right)$ characteristics for CG MESFFET and RDRP-CG MESFET under the gate bias varied from 0 to $12 \mathrm{~V}$ in a step of $-3 \mathrm{~V}$. As is shown in Figure II, the drain saturation current of RDRP-CG MESFET is about $582.6 \mathrm{~mA} / \mathrm{mm}$ in comparison with $520.6 \mathrm{~mA} / \mathrm{mm}$ of $\mathrm{CG}$ MESFET. There is about an $11.9 \%$ increase in drain saturation current.

One of the reasons is a thicker channel thickness in RDRPCG MESFET structure. The drain current is in direct proportion with the product of the thickness and the doping concentration of the channel $(\mathrm{N} \times \mathrm{a})$. Recessed $\mathrm{p}$-buffer layer of RDRP-CG MESFET makes the channel thickness thicker than CG MESFET. So the charge of channel increases, and then the current flow the channel increases, too. It means that the drain current increases. In addition, the extension of depletion region is weakened by recessed drain drift region, which makes the depletion region smaller than that of CG MESFET.
The smaller depletion region ensures that there is enough space for the charge to flow in the channel. As a result, the drain current of RDRP-CG MESFET can be enhanced compared to CG MESFET.

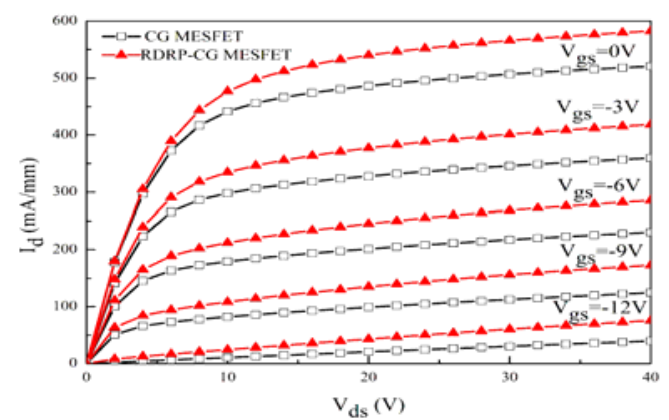

FIGURE II. SIMULATED DRAIN CURRENT-VOLTAGE (Id-Vds) CHARACTERISTICS UNDER DIFFERENT GATE VOLTAGES

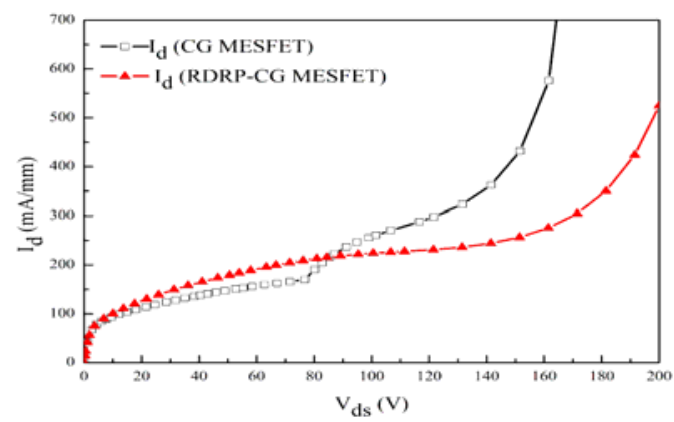

FIGURE III. SIMULATED BREAKDOWN CHARACTERISTICS FOr CG MESFET AND RDRP-CG MESFET

Figure III shows the simulated breakdown characteristics for CG MESFET and RDRP-CG MESFET at applied $\mathrm{V}_{\mathrm{gs}}=\mathrm{V}_{\mathrm{t}}$, which shows the drain current with respect to the drain-source voltage. It can be seen that the breakdown voltages for two structures are $76.6 \mathrm{~V}$ and $116.3 \mathrm{~V}$, respectively. It means that the breakdown voltage of RDRP-CG MESFET is increased by $51.8 \%$ than that of CG MESFET.

Electric Field distribution of CG MESFET and RDRP-CG MESFET from device left end to right end at $\mathrm{V}_{\mathrm{ds}}=\mathrm{V}_{\mathrm{b}}$ are shown in Figure IV. It is a special introduction to illustrate the reason of the increase in breakdown voltage. It can be noted that the highest electric field peak is at the gate corner near to drain side for both structures, that is to say the breakdown happens at gate corner near to drain side because of the electric field crowding effect at that corner [7,8]. It can be observed from Figure IV that other two electric field peaks are introduced in the proposed structure. One is at the corner of recessed drain drift region near to the drain side. The other one is at the edge of drain side. The reason in detail for this is that the recessed drain drift region make the channel between gate and drain thinner, and modulate the electric field in the channel, which get the electric field distribution in the channel 
more even. The even electric field distribution weakens the electric field crowding effect at gate corner. Therefore, a higher voltage is needed for the device to get its critical electric field. It leads to enhancing the breakdown voltage [9]. This is well validated by Figure IV.

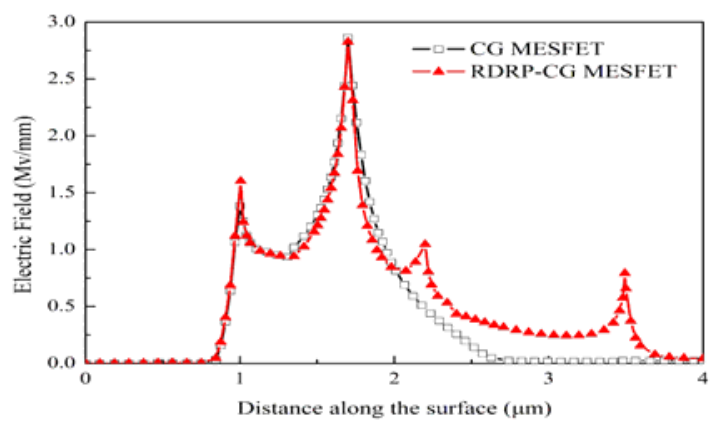

FIGURE IV. ELECTRIC FIELD DISTRIBUTION OF CG MESFET AND RDRP-CG MESFET FROM DEVICE LEFT END TO RIGHT END AT $\mathrm{Vds}=\mathrm{Vb}$

The maximum theoretical output power density $\left(\mathrm{P}_{\max }\right)$ for a class $\mathrm{A}$ amplifier is given by the following equation [6]:

$$
P_{\max }=I_{d s a t} \times\left(V_{b}-V_{\text {knee }}\right) / 8
$$

where $\mathrm{V}_{\mathrm{knee}}$ is the knee voltage. According to above equation, there is almost a $70 \%$ increase in the maximum output power density by calculating the maximum output power density of CG MESFET and RDRP-CG MESFET, which are $4.11 \mathrm{~W} / \mathrm{mm}$ and $6.98 \mathrm{~W} / \mathrm{mm}$, respectively. In brief, the proposed structure in the paper has a prominent DC characteristic.

\section{B. RF Characteristics}

The simulated gate-source capacitance versus frequency for two structures in the condition of $\mathrm{V}_{\mathrm{gs}}=0 \mathrm{~V}$ and $\mathrm{V}_{\mathrm{ds}}=40 \mathrm{~V}$ are presented in FigureV. It can be noted that the gate-source capacitance of RDRP-CG MESFET is $0.497 \mathrm{pF} / \mathrm{mm}$ compared to $0.435 \mathrm{pF} / \mathrm{mm}$ of CG MESFET [10]. The increase in gatesource capacitance is because of the decrease in the thickness of the depletion region. In detail, the capacitance equation can be expressed as follows:

$$
C=\varepsilon_{s} \times A / d
$$

where $\mathrm{d}$ is considered as the distance between metal and the depletion region around them in semiconductor device [11]. As seen in Figure VI, the bottom of the depletion region of RDRPCG MESFET moves towards gate side. Thus, the thickness of the depletion region $d$ is smaller than that of CG MESFET. Further, gate-source capacitance increase.

Figure VII shows the simulated trans-conductance versus gate voltage under $\mathrm{V}_{\mathrm{ds}}=40 \mathrm{~V}$ and $\mathrm{V}_{\mathrm{ds}}=1 \mathrm{~V}$. There is an increase in trans-conductance at $\mathrm{V}_{\mathrm{ds}}=40 \mathrm{~V}$ and small absolute value of $\mathrm{V}_{\mathrm{gg}}$. For the proposed structure, trans-conductance is bigger than that of CG MESFET, which means the ability of gate to control the channel is better.

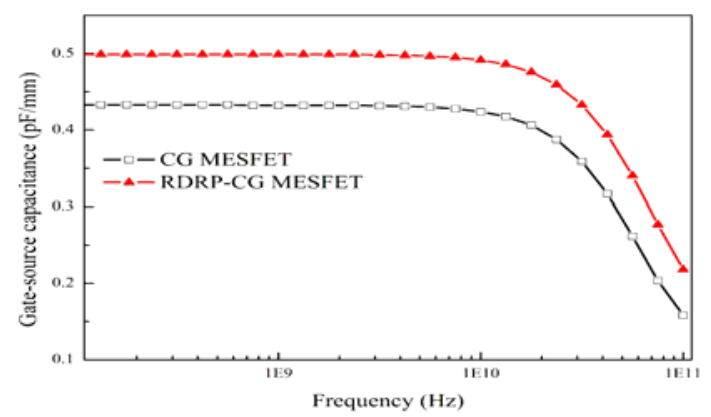

FIGURE V. SIMULATED GATE-SOURCE CAPACITANCE VERSUS FREQUENCY
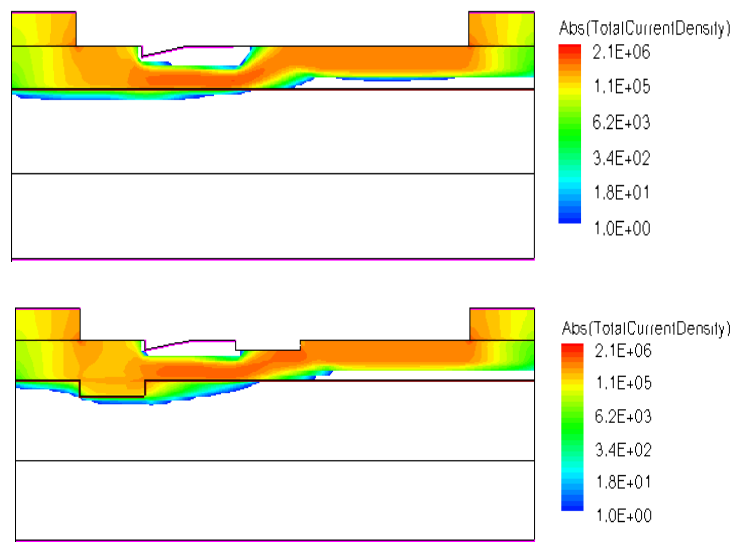

FIGURE VI. SIMULATED CURRENT DENSITY DISTRIBUTION OF CG MESFET AND RDRP-CG MESFET

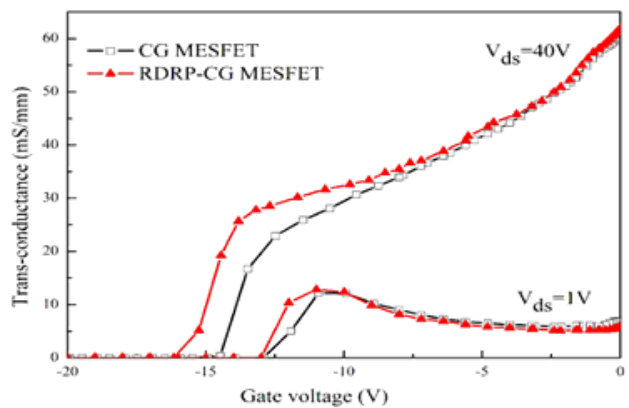

FIGURE VII. SIMULATED TRANS-CONDUCTANCE VERSUS GATE VOLTAGE UNDER Vds $=40 \mathrm{~V}$ AND Vds $=1 \mathrm{~V}$

All of those are benefit from the reduction in the equivalent resistance of channel. Compared to CG MESFET, the recessed region make the proposed structure has a smaller equivalent resistance of channel. When two structures are applied the same gate-source voltage in the case of a constant drain-source voltage, the proposed structure has a large transconductance. It is easier to control the channel well for RDRPCG structure. 
The cut-off frequency is connected with the ratio of transconductance and gate-source capacitance. According the discussion above, both trans-conductance and gate-source capacitance possess increases. The increase in gate-source capacitance is bigger than that in trans-conductance, which leads to a small decrease in cut-off frequency. The excellent characteristic in frequency for the proposed structure is not affected too much.

\section{CONCLUSION}

In this paper, the DC and RF characteristics of RDRP-CG MESFET have been simulated and compared to CG MESFET. Both drain saturation current and breakdown voltage are improved remarkably. The increases are $11.9 \%$ and $51.8 \%$, respectively. The evident rise in breakdown voltage should be noted specially, which means that RDRP-CG MESFET can work smoothly in high voltage environment. Due to the increase in drain current and breakdown voltage, the proposed structure gains a high output power density. All of these indicate the proposed structure has a superior DC characteristic. In terms of RF characteristics for the proposed structure, the enhancement of trans-conductance makes the gate bias control the channel more flexibly. The increase in trans-conductance of the proposed structure is smaller than that in gate-source capacitance, which results in a small reduction in cut-off frequency. By taking a panoramic view of the situation, the proposed structure gets admirable characteristics, which make it more suitable to RF system.

\section{ACKNOWLEDGMENT}

This work was supported by National Key Basic Research Program of China (973 Program) under grant No.2014CC339900, NSFC of China (No.61474089) and the Fundamental Research Funds for the Central Universities.

\section{REFERENCES}

[1] M. Franco, A. Katz. Class-E Silicon Carbide VHF Power Amplifier, Microwave Symposium, 2007. IEEE/MTT-S InternationalIEEE, 2007,pp.19-22.

[2] S.M. Noorbakhsh, H. Elahipanah, Improved 4H-SiC MESFET with double source field plate structures, Superlattices and Microstructures. vol.51(5), ,pp.553-562,2012.

[3] H. Elahipanah, Simulation and optimization of high breakdown doublerecessed $4 \mathrm{H}-\mathrm{SiC}$ MESFET with metal plate termination technique, Superlattices and Microstructures. vol.48(6) ,pp.529-540,2010.

[4] C.L. Zhu, C.C. Tin, G.H Zhang, S.F. Yoon, J. Ahn,, Improved performance of $\mathrm{SiC}$ MESFET using double-recessed structure, Microelectronic engineering. vol.83(1), pp.92-95,2006.

[5] H.J. Jia, D. Xing, H. Zhang, X.Y. Pei, Z.L. Sun, Y.C. Yuan, A novel 4HSiC MESFET with clival gate, Superlattices and Microstructures. vol.83, pp.29-34,2015.

[6] H.J. Jia, X.Y. Pei, Z.L. Sun, H. Zhang, Improved performance of $4 \mathrm{H}-$ silicon carbide metal semiconductor field effect transistors with multirecessed source/drain drift regions, Materials Science in Semiconductor Processing. vol.31, pp.240-244,2015.

[7] J. Zhang, X. Luo, Z. Li, et al. Improved double-recessed 4H-SiC MESFET structure with recessed source/drain drift region [J], Microelectronic Engineering. vol.84(12),pp.2888-2891,2007.

[8] X.C. Deng, B. Zhang, Y.R. Zhang, et al. Improved performance of $4 \mathrm{H}-$ $\mathrm{SiC}$ metal-semiconductor field-effect transistors with step p-buffer layer[J]. Chinese Physics B,vol. 20(1), 2011.
[9] Z. Ramezani, A.A. Orouji, P. Keshavarzi, A novel double-recessed 4HSiC MESFET using scattering the electric field for high power and RF applications, Physica E: Low-dimensional Systems and Nanostructures. vol.59, pp.202-209,2014.

[10] H.J. Jia, D. Xing, H. Zhang, et al. RF characteristics for $4 \mathrm{H}-\mathrm{SiC}$ MESFET with a clival gate[J]. Materials Science in Semiconductor Processing, vol.40, pp. 777-780,2015.

[11] A. Aminbeidokhti, A.A. Orouji, A novel 4H-SiC MESFET with modified channel depletion region for high power and high frequency applications, Physica E: Low-dimensional Systems and Nanostructures, vol.44(3), pp.708-713,2011. 